\title{
Muscle fatigue resistance in the rat hindlimb in vivo from low dietary intakes of tuna fish oil that selectively increase phospholipid $n-3$ docosahexaenoic acid according to muscle fibre type
}

\author{
R. Henry ${ }^{1}$, G. E. Peoples ${ }^{2,3}$ and P. L. McLennan ${ }^{2,3 *}$ \\ ${ }^{1}$ Exercise and Medical Science Division School of Medicine, University of Wollongong, Wollongong, NSW 2522, Australia \\ ${ }^{2}$ Graduate School of Medicine, University of Wollongong, Wollongong, NSW 2522, Australia \\ ${ }^{3}$ School of Medicine, Centre for Human and Applied Physiology, University of Wollongong, Wollongong, NSW 2522, Australia
}

(Submitted 24 February 2015 - Final revision received 8 May 2015 - Accepted 9 June 2015 - First published online 12 August 2015)

\section{Abstract}

Dietary fish oil (FO) modulates muscle $\mathrm{O}_{2}$ consumption and contractile function, predictive of effects on muscle fatigue. High doses unattainable through human diet and muscle stimulation parameters used engender uncertainty in their physiological relevance. We tested the hypothesis that nutritionally relevant FO doses can modulate membrane fatty acid composition and muscle fatigue. Male Sprague-Dawley rats were randomised to control (10\% olive oil (OO) by weight) or low or moderate FO diet (LowFO and ModFO) (HiDHA tuna fish oil) for 15 weeks (LowFO: 0.3\% FO, $9.7 \%$ OO, $0.25 \%$ energy as EPA + DHA; ModFO: $1.25 \%$ FO, $8.75 \%$ OO, 1.0 \% energy as EPA + DHA). Hindlimb muscle function was assessed under anaesthesia in vivo using repetitive $5 \mathrm{~s}$ burst sciatic nerve stimulation $(0 \cdot 05 \mathrm{~ms}, 7-12 \mathrm{~V}, 5 \mathrm{~Hz}, 10 \mathrm{~s}$ duty cycle, $300 \mathrm{~s}$ ). There were no dietary differences in maximum developed muscle force. Repetitive peak developed force fell to $50 \%$ within 62 (SEM 10) s in controls and took longer to decline in FO-fed rats (LowFO 110 (SEM 15) s; ModFO 117 (sEm 14) s) $(P<0 \cdot 05$ ). Force within bursts was better sustained with FO and maximum rates of force development and relaxation declined more slowly. The FO-fed rats incorporated higher muscle phospholipid DHA-relative percentages than controls $(P<0.001)$. Incorporation of DHA was greater in the fast-twitch gastrocnemius (Control 9.3 (SEm 0.8) \%, LowFO 19.9 (sem 0.4), ModFO 24.3 (sEm 1.0)) than in the slow-twitch soleus muscle (Control 5.1 (sEm 0.2), LowFO 14.3 (sem 0.7), ModFO 18.0 (SEM 1.4)) ( $P<0.001$ ), which was comparable with the myocardium, in line with muscle fibre characteristics. The LowFO and ModFO diets, emulating human dietary and therapeutic supplement intake, respectively, both elicited muscle membrane DHA enrichment and fatigue resistance, providing a foundation for translating these physiological effects to humans.

Key words: DHA: Fish oil: Skeletal muscle: Heart: Membrane fatty acids: Fibre type: Muscle fatigue

The gradual decline in repetitive force development that defines muscle fatigue can be attenuated by exercise training or dietary modulation of carbohydrate intake to optimise glycogen storage and availability, but there are no other recognised physiological approaches to fatigue prevention. Increased membrane phospholipid incorporation of long-chain $n$-3 PUFA (LC $n$-3 PUFA) DHA (22: $6 n-3)$, obtained from the diet via fish or fish oil (FO), is associated with increased efficiency of oxygen utilisation in the heart independent of heart rate ${ }^{(1)}$ and improved cardiac work recovery after ischaemic stress ${ }^{(1,2)}$. Dietary FO is also associated with increased whole human body oxygen efficiency during exercise ${ }^{(3)}$, implicating modified skeletal muscle $\mathrm{O}_{2}$ consumption. In humans and other vertebrates, DHA is the most unsaturated fatty acid present in cell membranes, accounting for up to $5 \%$ of all phospholipid fatty acids in most tissues. However, skeletal muscle ${ }^{(4-7)}$ has a predisposition to greater DHA incorporation, well beyond its relative percentage among circulating fats $^{(4,8)}$. Skeletal muscle shares this propensity for concentrated phospholipid DHA incorporation with other highly excitable tissues (myocardium, brain and retina) ${ }^{(4,5,8-11)}$. These parallels in membrane fatty acid composition, together with certain shared physiological properties ${ }^{(12)}$, imply an important role for adequate intake of LC $n$-3 PUFA supporting striated muscle physiology ${ }^{(13)}$.

High dietary intakes of $\mathrm{FO}$ in the rat are associated with apparent resistance of contracting skeletal muscles to fatigue ${ }^{(14,15)}$. Earlier in vitro studies have suggested a role of essential fatty acids (both $n$-6 PUFA and n-3 PUFA) in maintaining skeletal muscle function, compared with animals fed an essential fatty aciddeficient $\operatorname{diet}^{(16)}$. In these and other animal and human studies, physiological effects of LC $n-3$ PUFA have largely been investigated in relation to high intakes of $\mathrm{FO}^{(7,13)}$, commonly ranging from 5 to over $10 \%$ of diet by weight in animals or 5 to $8 \mathrm{~g} / \mathrm{d}$ in humans. However, dose-response studies show that the rat

Abbreviations: AA, arachidonic acid; FO, fish oil; LA, linoleic acid; LC $n$-3 PUFA, long-chain $n$ - 3 PUFA; OO, olive oil; SR, sarcoplasmic reticulum.

* Corresponding author: P. L. McLennan, fax +6124221 4341, email petermcl@uow.edu.au 
responds to very small dietary intakes of FO with large changes in myocardial membrane DHA incorporation ${ }^{(17)}$. This suggests that physiological changes may be achieved with lower, nutritionally relevant dietary interventions.

Although few studies have already revealed the potential influences of LC $n-3$ PUFA on skeletal muscle function, the single twitch model of contraction used in those dietary studies ${ }^{(14,15)}$ or tetanic stimulation protocols in other fatigue studies may not best represent submaximal and usual muscle $\operatorname{activity}^{(18,19)}$. Moreover, the translation of results to human nutrition must be made cautiously, as the high doses of FO previously used $^{(3,14,15)}$ are well beyond what could be obtained in the human diet.

The present study used the in vivo muscle function model of autologous pump-perfused hindlimb in anaesthetised rats. We developed this model ${ }^{(20)}$ so that the experiments could be carried out at physiological temperature to avoid the widely accepted influence of temperature on fatigue development and at physiological blood flow and arterial oxygen content so that the muscle could be oxygenated at physiological levels throughout and not randomly subjected to hypoxia or ischaemia $^{(19,21)}$. The autologous pump perfusion also ensured that muscle blood flow was controlled independently of intra-experimental fluctuations or dietary influences on cardiac output or blood pressure ${ }^{(20)}$. For this study, we further incorporated a stimulation protocol for the sciatic nerve: gastrocnemius-soleus-plantaris muscle bundle designed as a more physiological model to mimic functional fatigue. Two FO doses, derived from the studies of Slee et $a l .{ }^{(17)}$, were chosen to best replicate human nutritional and therapeutic supplement equivalents, respectively. This study tested the hypothesis that membrane change produced by low intakes of FO would be associated with resistance to physiologically relevant muscle fatigue.

\section{Methods}

\section{Animals}

In total, eighteen adult male Sprague-Dawley rats were housed two per cage at $23-25^{\circ} \mathrm{C}$ on a 12 light $-12 \mathrm{~h}$ dark cycle at the University of Wollongong's Animal facility. Experiments were approved by the University of Wollongong Animal Care and Ethics Committee and were conducted according to the Australian Code of Practice for the Care and Use of Animals for Scientific Purposes (National Health and Medical Research Council; https://www.nhmrc.gov.au/guidelines-publications). The research was conducted according to the ARRIVE guidelines for reporting in vivo animal experiments ${ }^{(22)}$.

Rats were obtained at 7 weeks of age (Animal Resources Centre) and fed a standard laboratory chow before randomly allocating them to be fed one of the three pre-fabricated diets ad libitum for 15 weeks from 8 to 10 weeks of age. The diets were prepared as previously developed for similar animal feeding studies ${ }^{(15,17)}$. Based on the American Institute of Nutrition AIN 93M diet ${ }^{(23)}$, they contained a balanced mix of macronutrients and micronutrients to avoid any nutritional deficiencies. All diets contained $10 \%$ fat by weight $(100 \mathrm{~g} / \mathrm{kg})$. Two sources of fat were used in this study to produce a control diet (10\% olive oil (OO)), low FO (LowFO) diet ( $0.31 \%$ FO, $9.7 \%$ OO) or a moderate FO (ModFO) diet ( $1.25 \%$ FO, $8.75 \%$ OO). OO was provided as 'extra light' OO, being largely devoid of the antioxidant polyphenols found in less-refined oils. OO consisted principally of oleic acid (18: 1, 75\%) and provided a minimum concentration of linoleic acid (LA) $(18: 2 n-6)$ to avoid essential fatty acid deficiency ${ }^{(2)}$. The FO was provided as HiDHA tuna fish oil (Nu-Mega Lipids), containing 29\% DHA and $7 \%$ EPA, $20 \%$ palmitic acid (16:0) and 14\% oleic acid as the major fatty acids. The fatty acid profiles of the control and FO diets are provided in Table 1 . The energy intake from these

Table 1. Dietary fatty acid composition for diets with different concentrations of fish oil (FO)

\begin{tabular}{|c|c|c|c|}
\hline & \multicolumn{3}{|c|}{ Diet } \\
\hline & Control & LowFO & ModFO \\
\hline FO (g/kg diet) & 0 & $3 \cdot 1$ & $12 \cdot 5$ \\
\hline $\mathrm{OO}$ ( $\mathrm{g} / \mathrm{kg}$ diet) & 100 & $96 \cdot 9$ & 87.5 \\
\hline \multicolumn{4}{|l|}{ Fatty acid $(\mathrm{g} / \mathrm{kg})$} \\
\hline $14: 0$ & 0 & 0.1 & 0.4 \\
\hline $16: 0$ & $10 \cdot 4$ & $10 \cdot 7$ & 11.6 \\
\hline $18: 0$ & $2 \cdot 8$ & $2 \cdot 9$ & $3 \cdot 1$ \\
\hline $18: 1(\mathrm{OA})$ & $75 \cdot 8$ & 73.9 & $68 \cdot 2$ \\
\hline $18: 2 n-6$ (LA) & $8 \cdot 3$ & $8 \cdot 1$ & 7.5 \\
\hline $18: 3 n-3$ (LNA) & 0.5 & 0.5 & 0.5 \\
\hline $20: 4 n-6$ (AA) & 0.1 & 0.2 & 0.3 \\
\hline $20: 5 n-3$ (EPA) & 0 & 0.2 & 0.9 \\
\hline $22: 5 n-3$ (DPA) & 0 & $0 \cdot 1$ & 0.1 \\
\hline $22: 6 n-3(\mathrm{DHA})$ & 0 & 0.9 & $3 \cdot 6$ \\
\hline$\Sigma n-6$ PUFA & $8 \cdot 7$ & 8.5 & 8.0 \\
\hline$\Sigma n-3$ PUFA & 0.5 & 1.7 & $5 \cdot 1$ \\
\hline$n-6: n-3$ PUFA ratio & $16 \cdot 63$ & 5.05 & 1.56 \\
\hline LA (\%en) & 1.92 & 1.87 & 1.72 \\
\hline EPA (\%en) & 0 & 0.05 & 0.20 \\
\hline DHA (\%en) & 0 & 0.20 & 0.83 \\
\hline Total fat (\%en) & 23.08 & 23.08 & 23.08 \\
\hline
\end{tabular}

Control diet, 0\% FO; LowFO, $0.31 \%$ low FO diet; ModFO, $1.25 \%$ moderate FO diet; OO, olive oil; OA, oleic acid; LA, linoleic acid; LNA, linolenic acid; AA, arachidonic acid. 
diets, based on a $300 \mathrm{~g}$ rat eating $20 \mathrm{~g} / \mathrm{d}$, was approximately $325 \mathrm{~kJ} / \mathrm{d}$. The LowFO diet $(3 \cdot 1 \mathrm{~g} / \mathrm{kg})$ was selected to emulate a human dietary EPA+DHA intake of $0.24 \%$ energy calculated equivalent to a human intake of $570 \mathrm{mg} / \mathrm{d} \mathrm{EPA}+\mathrm{DHA}$ and is a dose that approximately doubles myocardial DHA-relative percentage $^{(17)}$. The ModFO diet $(12.5 \mathrm{~g} / \mathrm{kg})$ was selected to emulate a human therapeutic supplement EPA + DHA intake of $1.0 \%$ energy equivalent to $2 \cdot 3 \mathrm{~g} / \mathrm{d} \mathrm{EPA}+\mathrm{DHA}$ and is a dose that produces changes in $n-3$ PUFA relative percentage that approach the asymptote of the dose-response curve for $n$-3 PUFA incorporation in cardiac ${ }^{(8,17)}$ and skeletal muscles ${ }^{(5)}$. This ModFO dose, already shown to be cardioprotective, is the lowest dose that has been tested physiologically to date ${ }^{(24)}$.

\section{Surgical hindlimb preparation}

The in vivo autologous, pump-perfused rat hindlimb preparation used in the present study has been described in detail and validated physiologically ${ }^{(20,25)}$. In brief, rats were anaesthetised (pentobarbitone sodium, $60 \mathrm{mg} / \mathrm{kg}$ i.p.) and maintained throughout the experiment with supplementary injections of $20 \mathrm{mg} / \mathrm{kg}$ i.p. pentobarbitone sodium. Rat body temperature was maintained at $37^{\circ} \mathrm{C}$ with the aid of a heated perspex chamber maintained at $31(\operatorname{SEM} 1)^{\circ} \mathrm{C}$ and an ancillary radiant heat lamp. Animals were ventilated at 60 breaths $/ \mathrm{min}$, and systemic blood pressure was monitored via the carotid artery. The left sciatic nerve was isolated for electrical stimulation of the hindlimb and the gastrocnemius-soleus-plantaris muscle bundle was attached to a force transducer (FT03C; Grass Technologies) at the Achilles tendon. A pump-perfusion system supplied the hindlimb muscles of the left leg below the knee with oxygenated arterial blood ${ }^{(20)}$. The femoral vein was cannulated for post-muscle blood sampling with venous blood returned passively to the systemic circulation. The extracorporeal perfusion circuit was primed with $6 \%$ dextran $(\mathrm{w} / \mathrm{v})$ (dextran 60; Sigma-Aldrich) and $5000 \mathrm{IU}$ heparin/100 ml (Sigma-Aldrich) in normal saline. The extracorporeal circuit plus the perfused muscle held a total volume of approximately $3 \mathrm{ml}$.

\section{Blood flow and muscle stimulation}

Once all cannulations were in place and blood flowed freely throughout the system, the pump was engaged to perfuse the left leg at $1 \mathrm{ml} / \mathrm{min}$, supporting resting blood flow requirements ${ }^{(20)}$, for $30 \mathrm{~min}$ equilibration before stimulating muscle contraction. Hindlimb perfusion pressure was monitored via a pressure transducer (Argon CDXIII; Maxim Medical) distal to the pump. The sciatic nerve was stimulated at supramaximal voltage to elicit muscle contraction and the muscle stretched to the optimal length for maximal twitch contraction force. Perfusion flow was increased to $1.5 \mathrm{ml} / \mathrm{min}$ to support the increased oxygen demand $^{(20)}$ for the duration of the muscle contraction protocol. Contractions were evoked using $5 \mathrm{~s}$ burst trains of pulses $(5 \mathrm{~Hz}, 7-12 \mathrm{~V}, 0.05 \mathrm{~ms}$ ) with $10 \mathrm{~s}$ duty cycle for $5 \mathrm{~min}$ (Fig. 1).

\section{Muscle samples and fatty acid analysis}

Gastrocnemius and soleus muscles were separated from the contralateral, unstimulated hindlimb during surgical preparation



Fig. 1. Typical experimental trace from the contracting rat hindlimb illustrating force development during a 5 -min stimulation protocol $(5 \mathrm{~Hz}, 5 \mathrm{~s}$ duty cycle) with (inset) one $5 \mathrm{~s}$ burst expanded to show the key derived measures of force and rate of force development $\left( \pm d T / d t_{\max }\right)$.

of the perfused limb, and the left ventricle was collected on completion of the hindlimb stimulation protocol, after euthanasia by rapid exsanguination under anaesthesia. Samples were rapidly taken from three sites: the left ventricle free wall; the entire soleus muscle cleared of connective tissue; and the lateral superficial gastrocnemius muscle belly. All samples were rapidly frozen and stored at $-80^{\circ} \mathrm{C}$. Skeletal and cardiac muscle samples (100$200 \mathrm{mg}$ ) underwent total lipid extraction using a modification of the Folch method ${ }^{(26)}$. Phospholipids were isolated from the total muscle lipid by solid-phase extraction using silica Sep-pak ${ }^{\mathrm{TM}}$ cartridges (Waters). Fatty acid methyl esters were prepared by direct transesterification ${ }^{(27)}$ of phospholipids and analysed by $\mathrm{GC}^{(17)}$ using a Shimadzu GC-17A (Shimadzu Australasia) with flame ionisation detection. Individual fatty acid peaks on the chromatogram were identified by comparison with authentic fatty acid methyl ester standards (Sigma-Aldrich Corporation) and $\mathrm{Nu}$ Chek Prep Inc. and expressed as percentage of total fatty acids in the phospholipid fraction. Peroxidisability index was calculated as the sum of bis-allelic hydrogen atoms (located on the methylene carbon atoms between two double bonds) according to the following formula: $(\%$ dienoic acids $\times 1)+(\%$ trienoics $\times 2)+(\%$ tetraenoics $\times 3)+(\%$ pentaenoics $\times 4)+(\% \text { hexaenoics } \times 5)^{(28)}$.

\section{Data analysis and calculations}

Contractile force was recorded and contraction characteristics were analysed using LabView for Windows with custom programming. Force and contraction characteristics were analysed for the 1st and the 25th (last) contraction in each $5 \mathrm{~s}$ contraction burst (Fig. 1). Fatigue was recorded as follows: (a) as the decline in developed force from 1st to 25 th contraction within each burst; and (b) as the decline in force between contraction bursts over time. The rate of fatigue between bursts was determined, from both 1 st and 25th contractions in each burst, as the time taken for the individual contraction-developed force to decline to $50 \%$ of the maximum peak contraction force. 


\section{Statistics}

Researchers were blinded to the allocation of animals to dietary groups until data collection and analysis were completed. Sample size calculation for membrane fatty acids estimated $n 4$ to detect $25 \%$ relative change in DHA ( $80 \%$ power for dose, $\alpha=0.05$, mean $\left.7.69 \%, \mathrm{SD}=1 \cdot 35 \%^{(17)}\right)$ and for contractile function, based on a $2 \mathrm{~Hz}$ continuous stimulation protocol, $n 5$ was required to detect $35 \%$ increase in time to fatigue ( $80 \%$ power, $\alpha=0.05$, mean $522 \mathrm{~s}, \mathrm{sD}=142 \mathrm{~s}$ ). Results are expressed as mean values with their standard errors of the mean. Two-way repeated measures ANOVA was used to analyse betweenmuscle-type differences and effects of diet on fatty acid incorporation into muscle tissues, with tissue and diet main effects and (diet $\times$ tissue) interaction, followed by Tukey's post hoc comparison of means (Statistix for Windows; Analytical Software). Statistical significance was accepted at $P<0.05$.

\section{Results}

\section{Effects of diet on body weight and muscle weight}

One rat was lost to the experiment due to excessive blood loss during preparative surgery, with no experimental data obtained, leaving the final numbers as follows: Control $n 6$; LowFO $n$; ModFO $n 7$ for all measures. After 15 weeks of dietary intervention, there were no significant dietary differences in body mass (Control: 463 (sem 30) g, LowFO: 457 (sem 44) g, ModFO: 464 (SEM 12) g) ( $P>0.05$ ); gastrocnemius-soleus-plantaris mass (Control: 3.02 (sem 0.12) g, LowFO: 3.15 (sem 0.15) g, ModFO: 3.03 (SEM 0.09) g) $(P>0.05)$ or in the ratio of gastrocnemiussoleus-plantaris mass:tibia length (Control: 6.91 (SEM 0.24), LowFO: 7.30 (SEM 0.24), ModFO: 6.89 (sem 0.15)) ( $P>0.05$ ).

\section{Effect of muscle type on membrane phospholipid fatty acid composition}

Statistically significant, between-tissue differences were observed for most fatty acids. In control animals, the few exceptions were as follows: no between-tissues differences in the minor LC $n$-3 PUFA, DPA ( $<1 \%$ ) or EPA (not detected) or in LA (18: $2 n-6)$, which was the most abundant individual fatty acid at about $20 \%$ of the total. There were no differences in membrane peroxidisability index between tissues in control animals (Table 2)

In control animals, the gastrocnemius muscle had significantly lower percentages of the SFA stearic acid 18 : 0 and MUFA oleic acid 18:1 compared with either soleus or myocardium, which were not significantly different to each other. In contrast, gastrocnemius had significantly higher percentages of the SFA palmitic acid $16: 0$ and LC $n$-3 PUFA DHA, as well as lower ratio of $n-6: n-3$ PUFA, than either the soleus or myocardium, which were not significantly different to each other (Table 2). In the soleus and myocardium, the principal SFA 18: 0 was found at twice the percentage of 16 : 0 , whereas in gastrocnemius the two were approximately equal. There were significant variations with tissue type in total relative percentages of SFA (gastrocnemius $>$ soleus, myocardium), MUFA (soleus $>$ myocardium, gastrocnemius) and PUFA (myocardium $>$ gastrocnemius, soleus).

With respect to LC PUFA (Table 2), the gastrocnemius muscle exhibited greater 22 : 6n-3 DHA and total n-3 PUFA and exhibited lower $20: 4 n-6$ arachidonic acid (AA), $18: 2 n-6$ LA, total $n$-6 PUFA percentages and $n-6: n-3$ PUFA ratio, compared with either the soleus or the left ventricle (all $P<0 \cdot 001$ ). The soleus further differed from the left ventricle in having lower percentages of 20 : $4 n-6$ AA and total $n-6$ PUFA (all $P<0 \cdot 001$ ). The LC $n$-3 PUFA $20: 5 n$-3 EPA was not detectable in any of the muscle tissues from control animals.

\section{Effect of fish oil diets on membrane fatty acid composition}

There were significant effects of FO diets on both types of skeletal muscle and cardiac membrane composition (Table 2). The major effects of dietary FO were increased percentages of DHA (22: $6 n-3, P<0 \cdot 001)$ and reduced AA (20: $4 n-6$, $P<0 \cdot 001)$. Smaller changes were observed in other fatty acids, including reduced percentages of LA $(18: 2 n-6, P<0 \cdot 001)$ and a small but statistically significant incorporation of EPA $(20: 5 n-3$, $P<0.001)$. These changes resulted in increased relative percentage of total $n-3$ PUFA $(P<0.001)$ and peroxidisability index $(P<0.001)$ and decreased relative percentage of total $n-6$ PUFA $(P<0 \cdot 001)$ and $n$-6:n-3 PUFA ratio $(P<0 \cdot 001)$. The incorporation of $n-3$ PUFA in exchange for $n-6$ PUFA was greater in the gastrocnemius muscle (all $P<0 \cdot 05$, diet $\times$ tissue interaction).

In the gastrocnemius muscle, the higher dose ModFO diet was associated with significant further decrease in relative percentage of AA and increased DHA compared with the LowFO diet, with further reduced total $n-6$ PUFA and increased total $n$-3 PUFA $(P<0.05)$ (Table 2$)$. In the soleus muscle and myocardium, the ModFO diet was associated with a small but significant further lowering in the relative percentage of total $n$-6 PUFA $(P<0.05)$ compared with the LowFO diet but no significant differences in DHA, LA, AA or any other major individual fatty acid or total $n$-3 PUFA (Table 2).

\section{Force characteristics}

Maximum peak contractile force was reached within the first few $5 \mathrm{~s}$ bouts of $5 \mathrm{~Hz}$ stimulation. The 1st contraction in each repeated $5 \mathrm{~s}$ bout was characterised by a rapid decline from bout to bout over $2-2.5$ min to a plateau level, which was maintained over the final minutes of the protocol (Fig. 2(a)). The developed tension of the 25th (last) contraction in repeated bouts also declined rapidly from bout to bout over $1-1.5 \mathrm{~min}$ to a plateau level maintained over the final minutes of the protocol (Fig. 2(b)). Peak developed force declined from contraction to contraction (1-25) within the $5 \mathrm{~s}$ bouts (Fig. 3).

There were no dietary differences in the maximum peak force (Control: 222 (sem 14); LowFO: 212 (sem 4); ModFO: 218 ( sem 9) N/100 g muscle mass) ( $P>0 \cdot 05)$. Peak developed force declined over time and significant diet $\times$ time interactions were evident (contraction 1: $P=0 \cdot 020$, contraction 25: $P<0 \cdot 001$ ). A significantly longer time was taken for the 1st contraction force to decline by $50 \%$ in the FO groups compared with control (Table 3) $(P<0.02)$ (Fig. 2(a)), and developed force was better sustained in the FO groups throughout and at the end of 
Table 2. Percentage of fatty acid composition of membrane phospholipids of heart and hindlimb muscles from rats after dietary fish oil (FO) supplementation for 15 weeks (Mean values with their standard errors; $n 4-6$ per group)

\begin{tabular}{|c|c|c|c|c|c|c|c|c|c|c|c|c|c|c|c|c|c|c|}
\hline \multirow{3}{*}{$\begin{array}{l}\text { Tissue } \\
\text { Diet }\end{array}$} & \multicolumn{6}{|c|}{ Gastrocnemius } & \multicolumn{6}{|c|}{ Soleus } & \multicolumn{6}{|c|}{ Left ventricle } \\
\hline & \multicolumn{2}{|c|}{ Control } & \multicolumn{2}{|c|}{ LowFO $(0.31 \%)$} & \multicolumn{2}{|c|}{ ModFO (1.25\%) } & \multicolumn{2}{|c|}{ Control } & \multicolumn{2}{|c|}{ LowFO $(0.31 \%)$} & \multicolumn{2}{|c|}{ ModFO (1.25\%) } & \multicolumn{2}{|c|}{ Control } & \multicolumn{2}{|c|}{ LowFO $(0.31 \%)$} & \multicolumn{2}{|c|}{ ModFO (1.25\%) } \\
\hline & Mean & SEM & Mean & SEM & Mean & SEM & Mean & SEM & Mean & SEM & Mean & SEM & Mean & SEM & Mean & SEM & Mean & SEM \\
\hline \multicolumn{19}{|l|}{ Fatty acid } \\
\hline $16: 0^{*}$ & $15 \cdot 70^{b}$ & 0.83 & $20 \cdot 39^{\mathrm{a}} \dagger$ & 0.97 & $21.98^{\mathrm{a}} \dagger$ & $2 \cdot 18$ & $9.83^{\mathrm{C}}$ & 0.56 & $15 \cdot 57^{\mathrm{b}} \dagger$ & 0.63 & $17.95^{\mathrm{a}} \dagger$ & 0.68 & $9.59^{c}$ & 0.18 & $11.57^{\mathrm{C}}$ & 0.21 & $12 \cdot 36^{b}$ & $0 \cdot 10$ \\
\hline $16: 1 \ddagger$ & $0.63^{\mathrm{a}}$ & 0.04 & $0.71^{a}$ & 0.08 & $0.88^{\mathrm{a}}$ & 0.17 & $0.75^{\mathrm{a}}$ & 0.09 & $0.68^{a, b}$ & 0.09 & $0.62^{\mathrm{a}}$ & 0.05 & $0.36^{\mathrm{b}}$ & 0.02 & $0.37^{\mathrm{b}}$ & 0.04 & $0.31^{b}$ & 0.02 \\
\hline $18: 0^{*}$ & $16 \cdot 15^{c}$ & 0.76 & $15 \cdot 83^{\mathrm{c}}$ & 0.58 & $15 \cdot 49^{c}$ & 0.66 & $18 \cdot 77^{\mathrm{b}}$ & 0.45 & $18 \cdot 17^{\mathrm{b}}$ & 0.65 & $19 \cdot 98^{\mathrm{a}}$ & 0.82 & $20 \cdot 49^{a}$ & 0.13 & $19.88^{a}$ & 0.24 & $20 \cdot 30^{\mathrm{a}}$ & 0.36 \\
\hline$\Sigma \mathrm{SFA}^{*}$ & $36 \cdot 40^{\mathrm{a}}$ & 1.06 & $38.84^{a}$ & 1.27 & $40 \cdot 30$ & 2.54 & $32.94^{b}$ & 0.73 & $35.79^{a, b}$ & 0.51 & $40.23 \dagger$ & 1.41 & $30 \cdot 11^{\mathrm{b}}$ & 0.12 & $33.46^{\mathrm{b}}$ & 0.13 & 34.91 & 0.30 \\
\hline $18: 1 \S$ & $10 \cdot 32^{\mathrm{b}}$ & 0.48 & $12 \cdot 81^{\mathrm{a}, \mathrm{b}} \dagger$ & 0.57 & $11.94^{\mathrm{b}} \dagger$ & 0.32 & $14.96^{a}$ & 1.08 & $15 \cdot 87^{\mathrm{a}}$ & 0.68 & $14 \cdot 19^{b}$ & 0.64 & $13 \cdot 43^{\mathrm{a}}$ & 0.21 & $11.26^{\mathrm{b}} \dagger$ & 0.20 & $10 \cdot 28^{\mathrm{b}} \dagger$ & 0.29 \\
\hline ¿MUFA§ & $12 \cdot 14^{\mathrm{b}}$ & 0.48 & $13.87^{a, b}$ & 0.61 & $13 \cdot 19$ & 0.50 & $17 \cdot 72^{\mathrm{a}}$ & 1.29 & $16 \cdot 99^{\mathrm{a}}$ & 0.64 & $15 \cdot 11$ & 0.62 & $13 \cdot 79^{\mathrm{b}}$ & 0.20 & $12 \cdot 06^{\mathrm{b}}$ & 0.20 & 11.01 & 0.31 \\
\hline $18: 2 n-6 \|$ & 19.66 & 0.85 & $16 \cdot 80$ & 0.74 & $15.09 \dagger$ & 1.03 & $20 \cdot 68$ & 0.55 & 18.78 & 0.54 & $17.59 \dagger$ & 0.26 & $21 \cdot 17$ & 0.39 & 19.25 & 0.71 & $18.08 \dagger$ & 0.32 \\
\hline $20: 3 n-6 \S$ & $0.78^{\mathrm{a}}$ & 0.03 & $0.51^{b} \dagger$ & 0.03 & $0.51^{b} \dagger$ & 0.04 & $0.80^{a}$ & 0.02 & $0.63^{\mathrm{a}} \dagger$ & 0.07 & $0.67^{\mathrm{a}}$ & 0.01 & $0.55^{\mathrm{b}}$ & 0.02 & $0.51^{\mathrm{b}}$ & 0.04 & $0.53^{\mathrm{b}}$ & 0.02 \\
\hline $20: 4 n-6^{*}$ & $15 \cdot 85^{\mathrm{b}}$ & 0.53 & $11.47^{\mathrm{b}} \dagger$ & 0.93 & $7.01^{b}+9$ & 1.06 & $16 \cdot 37^{\mathrm{b}}$ & 0.41 & $13 \cdot 61^{\mathrm{b}}+$ & 0.81 & $9 \cdot 36^{\mathrm{b}} \dagger$ & 0.61 & $24.04^{\mathrm{a}}$ & 0.37 & $18.41^{\mathrm{a}} \dagger$ & 0.45 & $15.65^{\mathrm{a}} \dagger$ & 0.55 \\
\hline $22: 4 n-6 \|$ & $0.39^{\mathrm{b}}$ & 0.09 & 0.19 & 0.05 & $0.03 \dagger$ & 0.03 & $0.66^{a}$ & 0.06 & $0.31 \dagger$ & 0.08 & $0.06+9$ & 0.06 & $0.70^{\mathrm{a}}$ & 0.04 & $0.32 \dagger$ & 0.03 & $0.12+\pi$ & 0.01 \\
\hline $22: 5 n-6 \ddagger$ & $1.39^{\mathrm{b}}$ & 0.11 & $0.35 \dagger$ & 0.04 & $0.40 \dagger$ & 0.01 & $1.24^{\mathrm{b}}$ & 0.08 & $0.41 \dagger$ & 0.05 & $0.23 t$ & 0.08 & $2 \cdot 07^{\mathrm{a}}$ & $0 \cdot 10$ & $0.62 \dagger$ & 0.04 & $0.57 \dagger$ & 0.03 \\
\hline$\Sigma n-6$ PUFA $^{*}$ & \multirow{2}{*}{\multicolumn{2}{|c|}{ ND }} & $28.99^{b} t$ & 0.99 & $22.97^{c}+9$ & 1.59 & \multirow{2}{*}{\multicolumn{2}{|c|}{ ND }} & $33 \cdot 27^{b} \dagger$ & 1.23 & $27 \cdot 72^{\mathrm{b}}+9$ & 0.81 & \multirow{2}{*}{\multicolumn{2}{|c|}{${ }^{40.34} \mathrm{ND}$}} & $38.83^{\mathrm{a}} \dagger$ & 0.56 & $34.88^{a}+9$ & 0.67 \\
\hline $20: 5 n-3$ & & & $0.16 \dagger$ & 0.04 & $0.36+9$ & $0 \cdot 10$ & & & 0.06 & 0.04 & $0.29 \dagger$ & 0.07 & & & 0.05 & 0.01 & $0.37+9$ & 0.04 \\
\hline $22: 5 n-3$ & $0.87^{\mathrm{a}}$ & 0.05 & 0.86 & 0.06 & $0.85^{\mathrm{b}}$ & 0.09 & $0.71^{\mathrm{b}}$ & 0.03 & $0.95 \dagger$ & 0.06 & $1.01^{a}+$ & 0.08 & $0.68^{\mathrm{b}}$ & 0.04 & 0.79 & 0.05 & $0.91^{\mathrm{b}} \dagger$ & 0.04 \\
\hline $22: 6 n-3 \|$ & $9 \cdot 26^{a}$ & 0.74 & $19.89^{\mathrm{a}} \mathrm{t}$ & 0.36 & $24 \cdot 25^{\mathrm{a}}+\pi$ & 1.03 & $5 \cdot 14^{\mathrm{b}}$ & 0.23 & $14 \cdot 27^{b} \dagger$ & 0.65 & $18.04^{\mathrm{b}} t$ & 1.40 & $6 \cdot 62^{b}$ & 0.34 & $16 \cdot 84^{\mathrm{b}} \dagger$ & 0.38 & $20.35^{\mathrm{b}} \dagger$ & 0.68 \\
\hline$\Sigma n-3$ PUFA $^{*}$ & $9.75^{a}$ & 0.63 & $18 \cdot 30^{a}+$ & 0.30 & $22 \cdot 33^{a}+\pi$ & 1.02 & $5 \cdot 62^{\mathrm{b}}$ & 0.21 & $13.35^{b} t$ & 0.63 & $16.93^{\mathrm{b}} t$ & 1.30 & $7 \cdot 30^{\mathrm{b}}$ & 0.34 & $15.65^{\mathrm{b}} \dagger$ & 0.35 & $19 \cdot 21^{b} t$ & 0.60 \\
\hline ¿PUFA & $51.45^{\mathrm{b}}$ & 0.89 & $47 \cdot 29^{b}$ & 0.76 & $45 \cdot 31^{\mathrm{b}}$ & $2 \cdot 16$ & $49 \cdot 34^{b}$ & 1.07 & $46 \cdot 62^{\mathrm{b}}$ & 1.36 & $44.66^{\mathrm{b}}$ & 1.81 & $56 \cdot 10^{\mathrm{a}}$ & 0.15 & $54.49^{\mathrm{a}}$ & 0.27 & $54.08^{a}$ & 0.47 \\
\hline$n-6: n-3$ ratioll & $4.33^{\mathrm{b}}$ & 0.34 & $1.59^{b}+$ & 0.08 & $1.03 \dagger$ & 0.07 & $7 \cdot 71^{\mathrm{a}}$ & 0.43 & $2.51^{\mathrm{a}} \dagger$ & 0.14 & $1.68 \dagger$ & 0.12 & $6 \cdot 72^{\mathrm{a}}$ & 0.34 & $2.49^{\mathrm{a}} \dagger$ & 0.09 & $1.83 \dagger$ & 0.09 \\
\hline PI§ & 136 & 3 & $156^{a}+$ & 2 & $162^{a}+$ & 7 & 125 & 1 & $143^{b}+$ & 4 & $147^{b}+$ & 7 & 134 & 2 & $166^{a}+$ & 2 & $173^{a}+$ & 2 \\
\hline
\end{tabular}

LowFO, low FO diet; ModFO, moderate FO diet; $\Sigma$ SFA, sum of SFA; $\Sigma$ MUFA, sum of MUFA; $\Sigma$ PUFA, sum of PUFA; PI, peroxidisability index (peroxidisability index was calculated from the formula: $(\%$ dienoic acids $\times 1)+(\%$

trienoics $\times 2)+(\%$ tetraenoics $\times 3)+(\%$ pentaenoics $\times 4)+(\%$ hexaenoics $\times 5))(28)$.
a,b,c Between tissues (within diets): mean values with unlike superscript letters were significantly different from those of other tissues within that diet.

Within tissues (between diets): $† P<0.05 \mathrm{v}$. control diet; $\uparrow P<0.05 \mathrm{v}$. LowFO diet. Overall between tissues (all diet groups combined): ${ }^{*} P<0.05 \mathrm{LV} \neq \mathrm{sol} \neq \mathrm{gastroc} ; \| P<0.05$ gastroc $\neq$ sol, LV; $\S P<0.05$ sol $\neq$ gastroc, LV; $\ddagger P<0.05$ $\mathrm{LV} \neq$ sol, gastroc 

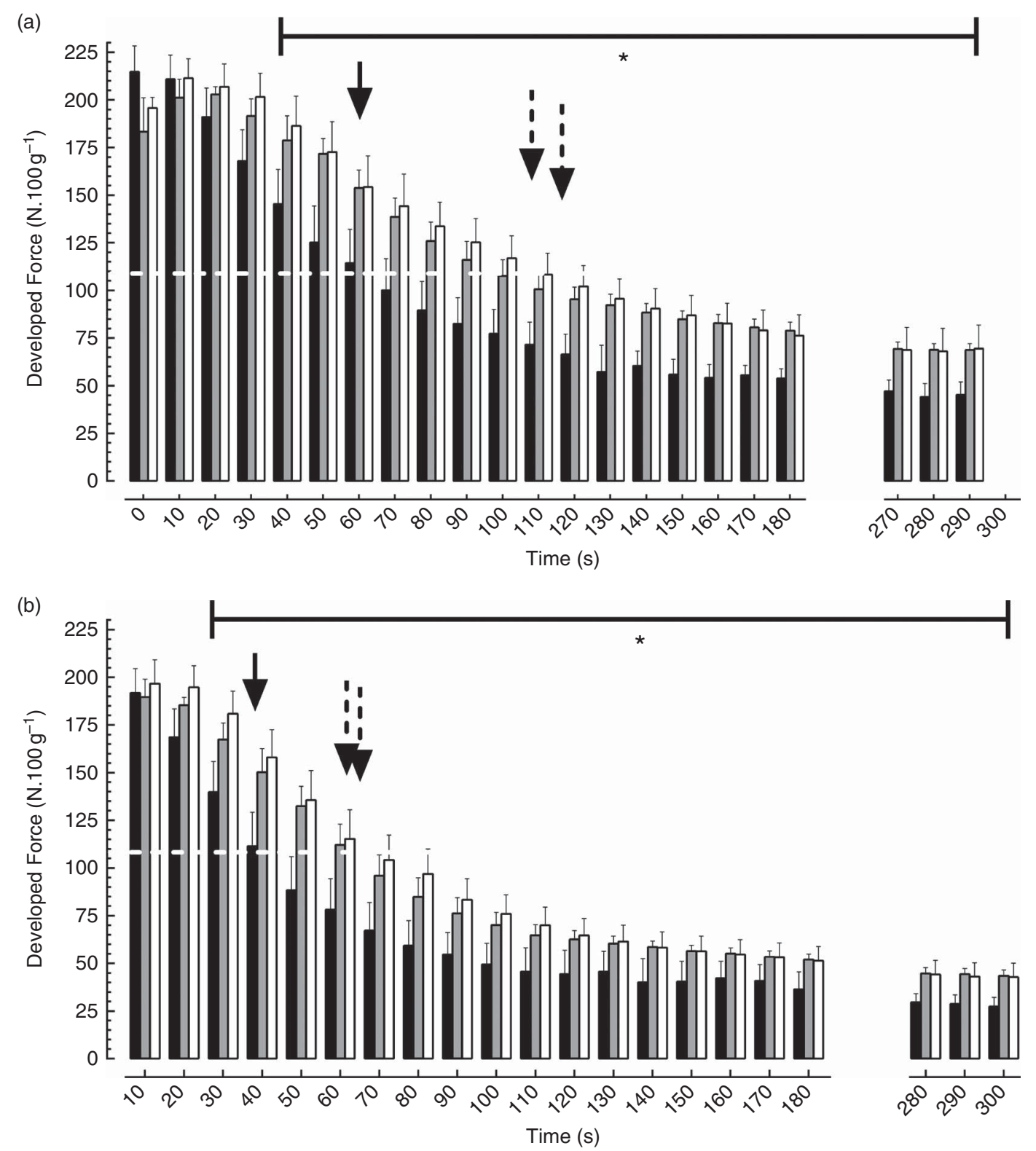

Fig. 2. Effect of diet on force production (N/100 g muscle mass) of gastrocnemius-soleus-plantaris muscle bundle during repeated burst $(5 \mathrm{~Hz}, 5 \mathrm{~s}$ duty cycle) stimulation for $5 \mathrm{~min}$. (a) Force of the 1 st contraction in each burst and (b) force of the 25 th (last) contraction in each burst. Horizontal broken lines represent $50 \%$ of maximum contraction (see Fig. 1 for illustration), arrows (solid = Control diet; broken = FO diets) show coincidence of the (a) 1st and (b) the 25th contraction in a burst with its decline to $<50 \%$ of the maximum. Bars represent mean values with their standard errors of the mean. Filled bars: Control diet $n 6$; shaded bars: LowFO diet $n$ 4; open bars: ModFO diet $n 7$. * LowFO, ModFO different from control $(P<0.05)$. FO, fish oil; LowFO, low FO diet; ModFO, moderate FO diet. $\square$, Control; $\square$, LowFO; $\square$, ModFO.

Table 3. Effect of dietary fish oil (FO) on time (s) to decline to $50 \%$ of the maximum for contraction and relaxation parameters of the 1st and the 25th (last) contractions in repetitive $5 \mathrm{~s}$ burst stimulation

(Mean values with their standard errors)

\begin{tabular}{|c|c|c|c|c|c|c|c|c|c|c|c|c|}
\hline \multirow[b]{3}{*}{ Diet } & \multicolumn{4}{|c|}{ Contraction peak force (s) } & \multicolumn{4}{|c|}{ Contraction rate $\left(+d T / d t_{\max }\right)(\mathrm{s})$} & \multicolumn{4}{|c|}{ Relaxation rate $\left(+d T / d t_{\max }\right)(\mathrm{s})$} \\
\hline & \multicolumn{2}{|c|}{ First } & \multicolumn{2}{|c|}{ Last } & \multicolumn{2}{|c|}{ First } & \multicolumn{2}{|c|}{ Last } & \multicolumn{2}{|c|}{ First } & \multicolumn{2}{|c|}{ Last } \\
\hline & Mean & SEM & Mean & SEM & Mean & SEM & Mean & SEM & Mean & SEM & Mean & SEN \\
\hline Control $(n 6)$ & 62 & 10 & 35 & 7 & 48 & 2 & 33 & 5 & 26 & 4 & 18 & 2 \\
\hline LowFO (n 4) & $110^{*}$ & 15 & $61^{*}$ & 8 & $94^{*}$ & 13 & $51^{*}$ & 8 & $35^{*}$ & 5 & 21 & 3 \\
\hline ModFO ( $n 7)$ & $117^{*}$ & 14 & $62^{*}$ & 11 & $96^{*}$ & 12 & $56^{*}$ & 9 & $37^{*}$ & 3 & $25^{*}$ & 3 \\
\hline
\end{tabular}

LowFO, low FO diet; ModFO, moderate FO diet.

${ }^{\star} P<0.05 v$. control diet 


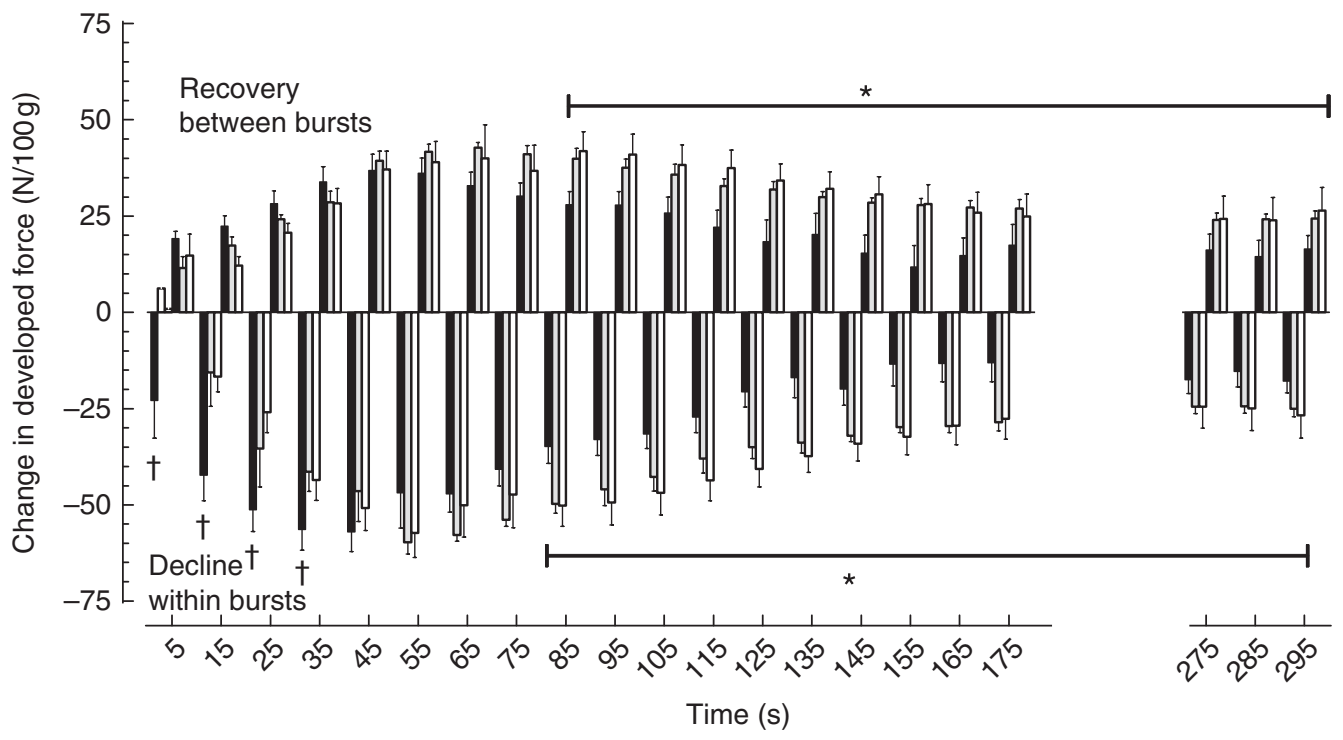

Fig. 3. Effect of diet on differences in developed force (N/100 g muscle mass) within bursts (below axis) and recovery between bursts (above axis) of ( $5 \mathrm{~Hz}, 5 \mathrm{~s}$ duty cycle) stimulation over $5 \mathrm{~min}$. Within-burst changes represent short-term fatigue over $5 \mathrm{~s}$. Between bursts represent recovery of contractile force in the $1 \mathrm{st}$ contraction of a new burst relative to the 25th (last) contraction of the previous one (see Fig. 1 for illustration). Bars represent mean values with their standard errors of the mean. Filled bars: Control diet $n 6$; shaded bars: LowFO diet $n 4$; open bars: ModFO diet $n 7$. ${ }^{*}$ LowFO, ModFO different from control $(P<0.05)$; $†$ LowFO, ModFO different from control $(P<0$.02). LowFO, low fish oil diet; ModFO, moderate fish oil diet. $\square$, Control; $\square$, LowFO; $\square$, ModFO.

the 5 min of contractions (Fig. 2) (contraction 1, contraction 25: $P<0 \cdot 001)$. There were no significant differences between LowFO and ModFO groups (contraction 1: $P=1 \cdot 000$, contraction 25: $P=0.993$ ).

Peak developed force declined within each $5 \mathrm{~s}$ burst (contractions 1-25), and over the first 60 s there was significantly less within-burst decline in the FO groups compared with control (Fig. 3). The FO groups took significantly longer for the 25 th contraction peak developed force to decline by $50 \%$ (Table 3 ) $(P<0.05)$. Maximum rates of force development and relaxation (Fig. 4) slowed down markedly over the first 60-100 s, and the decline in contraction and relaxation velocity was significantly greater in the control muscle than in FO muscles over the duration of the stimulation protocol. There were no significant differences between the LowFO and ModFO diets (Fig. 4).

\section{Hindlimb perfusion pressure and resistance}

There were no significant differences in resting hindlimb perfusion pressures across dietary groups (at $1 \mathrm{ml} / \mathrm{min}$ : Control 73 (sem 11) mmHg; LowFO 77 (sem 2); ModFO 76 (sem 4) and at $1.5 \mathrm{ml} / \mathrm{min}$ : Control 106 (SEM 13) mmHg; LowFO 109 (SEM 6); ModFO 104 (SEm 5)). Mean hindlimb perfusion pressure (at $1.5 \mathrm{ml} / \mathrm{min}$ ) decreased during muscle contractions in FO dietary groups $(P<0.05)$ with a non-significant trend in the control group ( $P=0.08$ ) (Control 92 (sem 13) mmHg; LowFO 85 (sem 3); ModFO 88 (SEM 4)). There were no significant differences in perfusion pressure between dietary groups at rest or during muscle activity $(P>0.05)$. A significantly greater percentage fall in mean hindlimb vascular resistance occurred during muscle contraction in the FO dietary groups compared with control $(P=0 \cdot 010)$.

\section{Discussion}

Membrane phospholipid fatty acid composition of rat skeletal muscle was highly responsive to dietary FO, and elevated incorporation of DHA was associated with resistance to muscle fatigue. This was achieved with a low-dose human nutritional equivalent of 1-2 fishmeals/week with little further dose-related changes, in either the membrane composition or the contraction parameters, obtained from the higher dose human therapeutic equivalent of $6-7 \mathrm{~g}$ of $\mathrm{FO} / \mathrm{d}^{(17)}$. The tuna fish oil supplement used in this study, with its high proportion of DHA, is consistent with the predominance of DHA in the human diet when obtained through common food fish ${ }^{(29)}$ and in contrast to most dietary FO supplements that commonly provide EPA:DHA in the ratio $180: 120 \mathrm{mg} / \mathrm{g}$. The in vivo hindlimb perfusion model permitted the examination of dietary effects on muscle function and fatigue under well-oxygenated, well-perfused and appropriate physiological conditions ${ }^{(20)}$. Effects on fatigue could be directly attributable to the change in muscle function without the potential confounding effects of dietary FO on cardiovascular function or behaviour. The membrane changes and fatigue resistance were achieved using much lower dietary LC $n$-3 PUFA concentrations than values previously reported in the literature. From these intakes, both of which were within a range that could reasonably be modulated nutritionally in man, skeletal muscle and myocardium incorporated high relative percentages of LC $n-3$ PUFA DHA into membrane phospholipids. Muscle types exhibited differences in fatty acid composition in accord with their contrasting physiological functions and fibre-type characteristics. Soleus muscle and myocardium had comparable patterns of fatty acid incorporation, including high DHA incorporation, in contrast to the fast twitch, fatigable gastrocnemius muscle, which incorporated even higher relative percentages of DHA. 

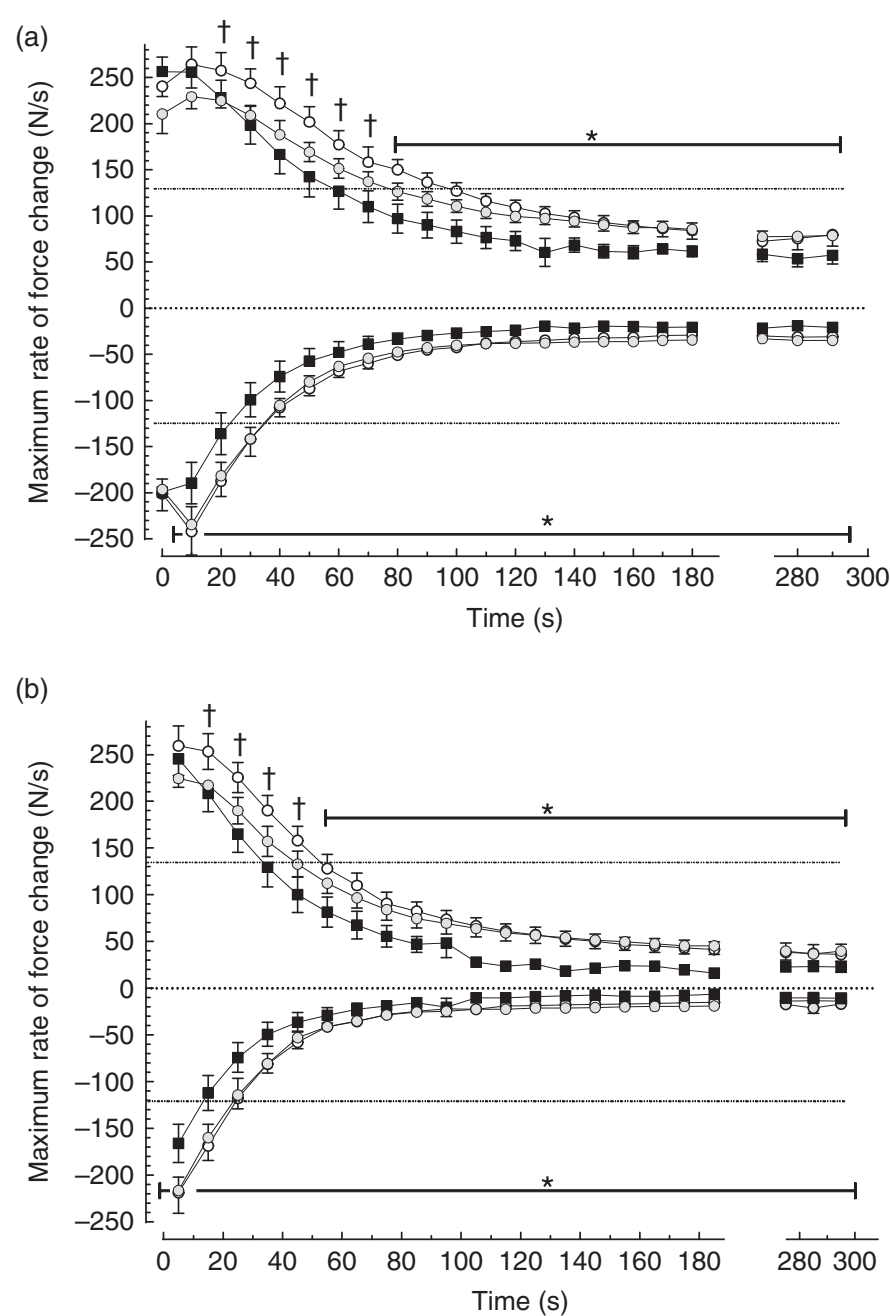

Fig. 4. Effect of diet on maximum rate of force development ( $d T / d t_{\max }$ above axis) and maximum rate of force decline ( $-d T / d t_{\text {max }}$ below axis) in individual contractions (N/100 g per s) during repeated burst $(5 \mathrm{~Hz}, 5 \mathrm{~s}$ duty cycle) stimulation for 5 min. (a) 1st contraction and (b) 25th (last) contraction in each $5 \mathrm{~s}$ burst (see Fig. 1 for illustration). Horizontal broken lines represent $50 \%$ of the maximum. Filled symbols: Control diet $n 6$; shaded symbols: LowFO diet $n 4$; open symbols: ModFO diet $n 7$. Symbols and bars represent mean values with their standard errors of the mean. ${ }^{*}$ LowFO, ModFO different from control $(P<0.05)$. $\dagger$ ModFO different from control $(P<0.02)$. LowFO, low fish oil diet; ModFO, moderate fish oil diet. $\longrightarrow$ - Control; $\longrightarrow$ — , LowFO; —o—, ModFO.

The lower of the two FO doses increased gastrocnemius and soleus muscle membrane DHA incorporation by $10 \%$ of the total fatty acids, despite providing $<1 \%$ of all the fatty acids in the diet. In contrast, LA diminished slightly in the membranes, despite being present at $8 \%$ of dietary fat and in an $n-6: n-3$ PUFA ratio of 5:1 in the lowFO diet, with the LA concentration almost ten times that of DHA. Concomitant reductions occurred in membrane AA of $<4 \%$ in skeletal muscle or $6 \%$ in the heart. Thus, skeletal muscle of the rat incorporated DHA into membrane phospholipids well above its relative percentage in the diet, as seen with high dietary FO doses ${ }^{(5)}$ and against an unfavourable ratio of $n-6: n-3$ PUFA. This finding confirms studies of skeletal muscle from developing rats and studies of rat myocardium that show that the absolute delivery of DHA is more important than its ratio to $n-6$ PUFA $^{(6,17)}$, even at these low intakes of LC $n$-3 PUFA. Although there can be no doubt that dietary $n$ - $6: n-3$ PUFA ratio powerfully influences $n$-3 PUFA membrane incorporation when it is dependent upon the shorter-chain precursor $\alpha$-linolenic acid $(18: 3 n-3)^{(30)}$, this is due to competition for enzyme sites for desaturation or elongation, limiting metabolic conversion to EPA and DHA. The present study demonstrated that the incorporation into membrane phospholipids is not subject to the same competition and confirms the previously reported lack of influence of dietary n-6 PUFA on LC $n$-3 PUFA incorporation into rat myocardium $^{(17)}$ or for clinical effect ${ }^{(31)}$. Striated muscle membranes preferentially incorporate DHA, and this is further illustrated by the very low incorporation of EPA into skeletal muscle or myocardium, despite significant dietary presentation in this study or when it is provided in a purified form in the $\operatorname{diet}^{(32)}$ or as high EPA FO that delivers plasma fatty acid EPA well in excess of $\mathrm{DHA}^{(8)}$. Some tissues such as platelets preferentially incorporate EPA, and the underlying physiological basis for differential incorporation is not known.

The slow-contracting, oxidative and fatigue-resistant soleus muscle, which provides slow-to-fatigue ankle stability and balance is in many ways similar to the ventricular myocardium. The soleus muscle and the myocardium share a common 
isoform of myosin heavy chain that exhibit low basal rates of ATP consumption (termed $\mathrm{MHC}_{\text {slow }}$ in type I muscle fibres and $\mathrm{MHC}_{\beta}$ in ventricular myocardium) ${ }^{(33)}$. The ATP reserve capacity associated with $\mathrm{MHC}_{\text {slow }}$ and the lower ATP cost for any developed tension in type I fibres ${ }^{(34)}$ confer ATP conservation and fatigue resistance on soleus muscle ${ }^{(35)}$. In contrast, in terms of fibre type, the portion of the gastrocnemius muscle sampled for fatty acid analysis in this study typically comprises mainly fast glycolytic/type IIb fibres with few of the slow-twitch, oxidative and fatigue-resistant type I fibres that almost exclusively make up the soleus muscle ${ }^{(36,37)}$. Type II fibres are characteristically glycolytic, fast-twitch and provide short-term power generation. They are rich in $\mathrm{MHC}_{2}$ isoforms ${ }^{(33)}$, which exhibit high rates of ATP consumption and low ATP reserve ${ }^{(34)}$, making them rapidly subject to fatigue with extended use. Preferential incorporation of DHA into the fast-contracting, powerful gastrocnemius muscle compared with the slower-contracting soleus has previously been reported in developing rats ${ }^{(6)}$. A similar very high incorporation of DHA into faster compared with slow muscle types is emphasised in species such as the rattlesnake and the hummingbird, which possess muscle groups of even more extreme contrast in contraction speed within them ${ }^{(38)}$. The higher retention of DHA in the gastrocnemius of non-supplemented rats may reflect an adaptive response to the higher maximum rates of ATP turnover of the largely fast-twitch gastrocnemius muscle fibres compared with the soleus and heart.

The dietary FO-induced proportional increases in membrane DHA increased the unsaturation and peroxidisability index of the muscle membranes, forecasting increased risk of oxidative damage $^{(28)}$ and fatigability ${ }^{(39)}$; however, this is contrary to what was borne out in the physiological measures of fatigue. Similarly, in the myocardium, the increase in peroxidisability induced by increased membrane DHA is paradoxically associated with reduced ischaemia-reperfusion fatty acid peroxidation and oxidative damage ${ }^{(28,40)}$. This has been attributed to an adaptive increase in the activity of superoxide dismutase and other endogenous antioxidant enzymes ${ }^{(40-42)}$. It suggests that chronically increased membrane peroxidisability induces chronic but non-damaging oxidative stress and adaptation in the same way that acute exercise induces oxidative stress, yet chronic exercise up-regulates antioxidant mechanisms and promotes fatigue resistance ${ }^{(43)}$. Alternatively, it was recently reported that anti-arrhythmic actions of DHA are enhanced by concomitant promotion of oxidation by $\mathrm{H}_{2} \mathrm{O}_{2}$ and inhibited by antioxidants, effects attributed to the specific non-enzymatic oxidative production of DHA-derived neuroprotectins ${ }^{(44)}$. Irrespective of the mechanism, increased peroxidisability of the membranes by enhanced DHA content is associated with protective rather than damaging effects under oxidative stress.

Non-dietary, adaptive increases in DHA incorporation and lower tissue $n$-6:n-3 PUFA ratio ${ }^{(45)}$ occur commonly in response to stresses in a variety of tissues including human exercising skeletal muscle ${ }^{(46)}$, ageing human heart ${ }^{(47)}$, human placenta at altitude ${ }^{(48)}$, chronic hypoxic rat heart ${ }^{(49)}$ and chronic catecholamine stress in rat heart ${ }^{(47)}$. An adaptive increase can be seen across mammalian species (including man) in relation to high BMR or resting heart rate, where the whale with its very low heart rate and high LC $n$-3 PUFA intake has very low myocardial DHA, in contrast to the mouse that has a very high heart rate, yet very high cardiac DHA-relative percentage, despite low LC $n$-3 PUFA intake ${ }^{(45,47)}$. In direct contrast, dietary intervention to raise cardiac DHA is associated with slower heart rates and preconditioning protection against these stresses $^{(2,13,45)}$. A similar observation applies to the restriction of acute muscle fatigue with dietary $n-3$ PUFA $^{(14,15)}$ in contrast to the preferential incorporation of DHA and lower $n-6: n-3$ PUFA ratio in the more rapidly fatiguing gastrocnemius ${ }^{(6)}$, and this may be related to the greater metabolic stress prevalent in type II fibres.

The upper dose ModFO (1.25\% FO) diet induced DHA incorporation in the gastrocnemius to the same extent as reported previously with high dose $7 \%$ FO supplementation in the similar, mixed fibre-type $m$. vastus lateralis $^{(14)}$. In the present study, there was little displacement of tissue LA compared with earlier studies that used high FO doses. With diets in those studies delivering six times the DHA dose and a $n-6: n-3$ PUFA ratio of $<0 \cdot 2$, it is evident that the previous studies used FO doses far in excess of requirements for maximal effect.

The modulation of rat muscle phospholipid fatty acid composition, as a result of these small FO intakes, induced marked resistance to muscle fatigue in vivo without influencing the initial peak force of contraction of the hindlimb. Fatigue in the contracting hindlimb could be characterised in terms of extent of decline in twitch force within the 25 contractions of any $5 \mathrm{~s}$ burst; extent of decline in twitch force from burst to burst (which has a recovery component); the time course of the decline in twitch force; the decline in maximum rate of force production and relaxation; and the time course of those changes. The dietary FO markedly attenuated the decrease in muscle force production and extended the time course of well-sustained isometric force production. In other words, the hindlimb muscles of animals fed FO were able to complete more contractions at a force closer to their peak isometric tension. In skeletal muscles, when force is sustained at a higher relative tension over time, it is explained by the optimal coupling relationship between cellular ATP demand, inherently the efficiency of ATP use by the contracting cell, and the metabolic supply ${ }^{(50,51)}$. Furthermore, although muscles sustained higher peak contractile force for longer after FO feeding, the attenuation in decline in rates of force production and relaxation also implies an optimal coupling of net $\mathrm{Ca}^{2+}$ turnover and cellular ATP maintenance, most importantly associated with the powerful but fatigable, fast-twitch fibres ${ }^{(52)}$.

Notably, the greatest effect of dietary FO, retarding fatigue, occurred in the earliest phase of contraction, which corresponds to the highest rate of tension decline from peak force. With single-pulse or tetanic burst contractions, fatigue occurs much more rapidly in fast, type II muscle fibres than in slow, type I fibres in shortening or isometric contractions ${ }^{(19,21)}$. This response is likely due to the greater part of the hindlimb muscle bundle representing fast, type II fibres, densely packed with sarcoplasmic reticulum (SR) and expressing high sarcoplasmic reticulum $\mathrm{Ca}^{2+}$ ATPase (SERCA) concentration, a requirement to sustain rapid force production and relaxation. Highly effective SERCA, such as those in the powerful muscle fibres, rely on the phospholipid environment to carry out rapid removal of calcium against its concentration gradient ${ }^{(53)}$. There is strong 
evidence that when DHA makes up a high proportion of the membrane fatty acids, this process of $\mathrm{Ca}^{2+}$ pumping is optimised $^{(38)}$, thus sustaining force production in the periods of rapid fatigue.

Although this study demonstrates a clear association between dietary fatty acids, muscle incorporation of DHA and fatigue resistance, it does not identify the mechanisms of fatigue that are affected. As FO feeding does not modify glycogen storage or attenuate metabolic acidosis during fatiguing muscle stimulation ${ }^{(15)}$, we can exclude the two most common interventional approaches used ahead of exercise to improve muscle function: promotion of glycogen storage and metabolic alkalosis inducible by sodium bicarbonate ingestion ${ }^{(54)}$. We must, therefore, consider one or more of the many other cellular mechanisms potentially underlying fatigue resistance ${ }^{(21,19)}$. Although they are not readily directly examinable in vivo ${ }^{(19)}$, some insight into the potential mechanisms of action of LC $n-3$ PUFA fatigue resistance may be gained from comparison with interventions that, in contrast to FO, enhance muscle fatigue.

The pattern of improved muscle function by FO relative to control contrasts with the effects that the $\beta_{2}$ adrenoceptor agonist clenbuterol has on muscle function. Chronic clenbuterol treatment has found some popularity in body building for its promotion of muscle hypertrophy, but it significantly slows relaxation and decreases resistance to fatigue in fast-twitch muscle fibres ${ }^{(55)}$. Clenbuterol's functional effects appear linked to intra-cellular $\mathrm{Ca}^{2+}$ homoeostasis, especially the leakage of SR $\mathrm{Ca}^{2+(55)}$. The decline in $\mathrm{SR} \mathrm{Ca}^{2+}$ and slow $\mathrm{SR} \mathrm{Ca}^{2+}$ re-uptake, with the latter contributing to slowed isometric relaxation, are believed to underpin fatigue in both fast- and slow-twitch muscles under tetanic ${ }^{(56)}$ or non-tetanic stimulation ${ }^{(57,58)}$. If FO were to prevent $\mathrm{SR} \mathrm{Ca}^{2+}$ leakage and promote SR $\mathrm{Ca}^{2+}$ re-uptake, this could explain the fatigue resistance. Indeed, such an effect has been observed in the myocardium in which altered $\mathrm{Ca}^{2+}$ handling is recognised to play a part in DHA action in myocardial intra-cellular signalling, with modulated SR $\mathrm{Ca}^{2+}$ leakage implicated in cardiac pacemaker slowing and arrhythmia prevention in cardiac muscles ${ }^{(13)}$. Both direct and indirect evidence shows increased efficiency of SR $\mathrm{Ca}^{2+}$ handling as a basis for the prevention of arrhythmia ${ }^{(1,44)}$ and reduced mitochondrial $\mathrm{Ca}^{2+}$ uptake as a basis for increased oxygen efficiency ${ }^{(1)}$ or as the basis for reduced mitochondrial pyruvate dehydrogenase activity ${ }^{(59)}$ in the rat myocardium after FO feeding. However, the evidence is equivocal with another study finding that dietary FO has little influence on cardiac SERCA activity and may even increase cardiac mitochondrial $\mathrm{Ca}^{2+}$-ATPase activity ${ }^{(60)}$. Moreover, an in vitro study of SR function of skeletal muscle from rats fed DHA also revealed reduced SERCA activity and increased $\mathrm{Ca}^{2+}$ leakage ${ }^{(61)}$, which would predict slower relaxation and increased energy requirements and fatigability, the opposite to what is actually observed in vivo during muscle contraction in the present study. Further studies are needed to investigate the role of altered $\mathrm{Ca}^{2+}$ homoeostasis on the effects that membrane incorporation of DHA has on muscle fatigue.

Further studies are also required to identify effects more specifically in fast- and slow-twitch fibre types in accordance with differences in DHA incorporation. The improved muscle function during the early fast-fatiguing component of the non-tetanic repetitive burst stimulation protocol implicates fast, type II fibres in a primary role in this study, in line with the predominance of the gastrocnemius muscle in the contracting bundle. However, slow type I fibres and involvement of the soleus muscle are also implicated in DHA effects by the sustained greater force and better sustained rates of force development observed after the early fatigue phase, as well as the continued greater recovery between contraction bouts after FO feeding. Although the myocardium typically does not fatigue acutely, contractile function and relaxation do decline significantly during heart failure and this can be counteracted by feeding $\mathrm{FO}^{(62,63)}$. The patterns of enhanced skeletal muscle fatigue in rats with heart failure ${ }^{(21)}$ suggest that a benefit could be gained by increasing membrane DHA.

This study demonstrates that marked changes in muscle membrane fatty acid composition together with resistance to muscle fatigue are achievable in rat skeletal muscle with only small dietary supplements of LC $n$-3 PUFA, in a range readily compatible with human nutrition ${ }^{(13)}$. The low effective dose and the DHA-rich tuna fish oil supplement replicate human dietary patterns achievable from a low-moderate intake of fish, and provide a basis for translating to humans the physiological observations made in this study and those previously made using high doses of FO in animals. Furthermore, tissue differences in membrane fatty acid composition suggest specific incorporation of fatty acids reflective of physiological function, with the fatigueresistant slower-contracting soleus muscle and myocardium exhibiting many compositional similarities in contrast with the highly fatigable fast-contracting gastrocnemius muscle. Of particular note, although it is generally incorporated well in all striated muscles, DHA was innately incorporated in higher relative percentages in the gastrocnemius compared with the soleus muscle and myocardium without any dietary intervention as well as in response to FO feeding, perhaps reflecting an adaptive response to the higher peak metabolic demand and fatigability of the predominant type II muscle fibres. The large changes in composition and function achieved with only small additions of FO to the diet suggest that DHA may be an essential component of striated muscle for optimal healthy function and that the failure to include regular fish or FO in the diet might lead to a deficiency reflected in susceptibility to muscle fatigue.

\section{Acknowledgements}

The authors thank Clover Corporation and Nu-Mega Lipids (Australia) for their generous donation of high-DHA tuna fish oil.

This research received no specific grant from any funding agency, commercial or not-for-profit sectors. The research was supported by the donation of high-DHA tuna fish oil from Clover Corporation and Nu-Mega Lipids (Australia).

R. H., G. E. P. and P. L. M. conceived and designed the research; R. H. collected and analysed the data; R. H. and P. L. M. statistically analysed the data; and R. H., G. E. P. and P. L. M. interpreted the data and provided important intellectual content for drafting the manuscript. P. L. M. had the primary responsibility for the final content.

There are no conflicts of interest. 


\section{References}

1. Pepe S \& McLennan PL (2002) Cardiac membrane fatty acid composition modulates myocardial oxygen consumption and post-ischemic recovery of contractile function. Circulation 105, 2303-2308.

2. Abdukeyum GG, Owen AJ \& McLennan PL (2008) Dietary (n-3) long-chain polyunsaturated fatty acids inhibit ischemia and reperfusion arrhythmias and infarction in rat heart not enhanced by ischemic preconditioning. J Nutr 138, 1902-1909.

3. Peoples GE, McLennan PL, Howe PRC, et al. (2008) Fish oil reduces heart rate and oxygen consumption during exercise. J Cardiovasc Pharmacol 52, 540-547.

4. Charnock JS, Abeywardena MY, Poletti VM, et al. (1992) Differences in fatty acid composition of various tissues of the marmoset monkey (Callithrix jacchus) after different lipid supplemented diets. Comp Biochem Physiol A Mol Integr Physiol 101, 387-393.

5. Owen AJ, Peter-Przyborowska BA, Hoy AJ, et al. (2004) Dietary fish oil dose- and time-response effects on cardiac phospholipid fatty acid composition. Lipids 39, 955-961.

6. Stark KD, Lim SY \& Salem N (2007) Docosahexaenoic acid and $n-6$ docosapentaenoic acid supplementation alter rat skeletal muscle fatty acid composition. Lipids Health Dis $\mathbf{6}, 13$.

7. Storlien LH, Jenkins AB, Chisholm DJ, et al. (1991) Influence of dietary fat composition on development of insulin resistance in rats. Relationship to muscle triglyceride and $n-3$ fatty acids in muscle phospholipids. Diabetes 40, 280-289.

8. McLennan PL (2001) Myocardial membrane fatty acids and the antiarrhythmic actions of dietary fish oil in animal models. Lipids 36, S111-S114.

9. Hock CE, Holahan MA \& Reibel DK (1987) Effect of dietary fish oil on myocardial phospholipids and myocardial ischemic damage. Am J Physiol 252, H554-H560.

10. Rocquelin G, Guenot L, Justrabo E, et al. (1985) Fatty acid composition of human heart phospholipids: data from 53 biopsy specimens. J Mol Cell Cardiol 17, 769-773.

11. Moriguchi T, Loewke J, Garrison M, et al. (2001) Reversal of docosahexaenoic acid deficiency in the rat brain, retina, liver, and serum. J Lipid Res 42, 419-427.

12. Wasserstrom JA (1998) New evidence for similarities in excitation-contraction coupling in skeletal and cardiac muscle. Acta Physiol Scand 162, 247-252.

13. McLennan PL (2014) Cardiac physiology and clinical efficacy of dietary fish oil clarified through cellular mechanisms of omega-3 polyunsaturated fatty acids. Eur J Appl Physiol 114, 1333-1356.

14. Peoples GE \& McLennan PL (2010) Dietary fish oil reduces skeletal muscle oxygen consumption, provides fatigue resistance and improves contractile recovery in the rat in vivo hindlimb. Br J Nutr 104, 1771-1779.

15. Peoples GE \& McLennan PL (2014) Long chain omega-3 docosahexaenoic acid reduces the extent of skeletal muscle fatigue in the rat in vivo hindlimb. Br J Nutr 111, 996-1003.

16. Ayre KJ \& Hulbert AJ (1996) Dietary fatty acid profile influences the composition of skeletal muscle phospholipids in rats. J Nutr 126, 653-662.

17. Slee EL, McLennan PL, Owen AJ, et al. (2010) Low dietary fish oil threshold for myocardial membrane $n$-3 PUFA enrichment independent of $n-6$ PUFA intake in rats. J Lipid Res 51, 1841-1848.

18. Jones DA (1996) High- and low-frequency fatigue revisited. Acta Physiol Scand 156, 265-270.

19. Allen DG, Lamb GD \& Westerblad H (2008) Skeletal muscle fatigue: cellular mechanisms. Physiol Rev 88, 287-332.

20. Peoples GE, Hoy AJ, Henry R, et al. (2013) Autologous pumpperfused rat hindlimb preparation for investigating muscle function and metabolism in vivo. Microcirculation 20, 511-523.
21. Lunde PK, Verburg E, Eriksen M, et al. (2002) Contractile properties of in situ perfused skeletal muscles from rats with congestive heart failure. J Physiol 540, 571-580.

22. Kilkenny C, Browne W, Cuthill IC, et al. (2010) Animal research: reporting in vivo experiments: the ARRIVE guidelines. Br J Pharmacol 160, 1577-1579.

23. Reeves PG (1997) Components of the AIN-93 diets as improvements in the AIN-76A diet. J Nutr 127, 838S-841S.

24. McLennan PL, Owen AJ, Slee EL, et al. (2007) Myocardial function, ischaemia and $n-3$ polyunsaturated fatty acids: a membrane basis. J Cardiovasc Med 8, Suppl. 1, S15-S18.

25. Hoy AJ, Peoples GE \& McLennan PL (2009) The effect of vasoconstrictors on oxygen consumption in resting and contracting skeletal muscle of the autologous pump-perfused rat hindlimb. J Physiol Pharmacol 60, 155-160.

26. Folch J, Lees M \& Sloane Stanley GH (1957) A simple method for the isolation and purification of total lipides from animal tissues. J Biol Chem 226, 497-509.

27. Lepage G \& Roy C (1986) Direct transesterification of all classes of lipids in a one-step reaction. J Lipid Res 27, 114-121.

28. Kubo K, Saito M, Tadokoro T, et al. (1998) Dietary docosahexaenoic acid dose [sic] not promote lipid peroxidation in rat tissue to the extent expected from peroxidizability index of the lipids. Biosci Biotechnol Biochem 62, 1698-1706.

29. Mozaffarian D \& Wu JHY (2011) Omega-3 fatty acids and cardiovascular disease effects on risk factors, molecular pathways, and clinical events. J Am Coll Cardiol 58, 2047-2067.

30. Tu WC, Muhlhausler BS, Yelland LN, et al. (2013) Correlations between blood and tissue omega-3 LCPUFA status following dietary ALA intervention in rats. Prostaglandins Leukot Essent Fatty Acids 88, 53-60.

31. Griffin BA (2008) How relevant is the ratio of dietary $n-6$ to $n-3$ polyunsaturated fatty acids to cardiovascular disease risk? Evidence from the OPTILIP study. Curr Opin Lipidol 19, 57-62.

32. McLennan P, Howe P, Abeywardena M, et al. (1996) The cardiovascular protective role of docosahexaenoic acid. Eur J Pharmacol 300, 83-89.

33. Sieck GC \& Regnier M (2001) Plasticity in skeletal, cardiac, and smooth muscle - invited review: plasticity and energetic demands of contraction in skeletal and cardiac muscle. J Appl Physiol 90, 1158-1164.

34. Han YS, Proctor DN, Geiger PC, et al. (2001) Reserve capacity for ATP consumption during isometric contraction in human skeletal muscle fibers. J Appl Physiol 90, 657-664.

35. Howlett RA \& Hogan MC (2007) Effect of hypoxia on fatigue development in rat muscle composed of different fibre types. Exp Physiol 92, 887-894.

36. Delp MD \& Duan C (1996) Composition and size of type I, IIA, IID/X, and IIB fibers and citrate synthase activity of rat muscle. $J$ Appl Physiol 80, 261-270.

37. Armstrong RB \& Phelps RO (1984) Muscle fibre type composition of the rat hindlimb. Am J Anat 171, 259-272.

38. Infante JP, Kirwan RC \& Brenna JT (2001) High levels of docosahexaenoic acid (22: $6 n$-3)-containing phospholipids in highfrequency contraction muscles of hummingbirds and rattlesnakes. Comp Biochem Physiol B Biochem Mol Biol 130, 291-298.

39. Powers SK \& Jackson MJ (2008) Exercise-induced oxidative stress: cellular mechanisms and impact on muscle force production. Physiol Rev 88, 1243-1276.

40. Erdogan H, Fadillioglu E, Ozgocmen S, et al. (2004) Effect of fish oil supplementation on plasma oxidant/antioxidant status in rats. Prostaglandins Leukot Essent Fatty Acids 71, 149-152.

41. Jahangiri A, Leifert WR, Kind KL, et al. (2006) Dietary fish oil alters cardiomyocyte $\mathrm{Ca}^{2+}$ dynamics and antioxidant status. Free Radic Biol Med 40, 1592-1602. 
42. Molinar-Toribio E, Pérez-Jiménez J, Ramos-Romero S, et al. (2015) Effect of $n-3$ PUFA supplementation at different EPA:DHA ratios on the spontaneously hypertensive obese rat model of the metabolic syndrome. Br J Nutr $\mathbf{1 1 3}$, 878-887.

43. Gomes EC, Silva AN \& de Oliveira MR (2012) Oxidants, antioxidants, and the beneficial roles of exercise-induced production of reactive species. Oxid Med Cell Longev 2012, Article ID 756132.

44. Roy J, Oger C, Thireau J, et al. (2015) Non-enzymatic lipid mediators, neuroprostanes, exert the anti-arrhythmic properties of docosahexaenoic acid. Free Radic Biol Med 86, 269-278.

45. McLennan PL \& Abeywardena MY (2005) Membrane basis for fish oil effects on the heart: linking natural hibernators to prevention of human sudden cardiac death. J Membr Biol 206, 85-102.

46. Helge JW, Wu BJ, Willer M et al. (2001) Training affects muscle phospholipid fatty acid composition in humans. J Appl Physiol 90, 670-677.

47. Gudbjarnason S (1990) What is the function of docosahexaenoic acid in heart-muscle membranes. J Appl Cardiol 5, $13-21$.

48. Tissot van Patot MC, Murray AJ, Beckey V, et al. (2010) Human placental metabolic adaptation to chronic hypoxia, high altitude: hypoxic preconditioning. Am J Physiol Regul Integr Comp Physiol 298, R166-R172.

49. Balkova P, Jezkova J, Hlavackova M, et al. (2009) Dietary polyunsaturated fatty acids and adaptation to chronic hypoxia alter acyl composition of serum and heart lipids. $\mathrm{Br} J \mathrm{Nutr}$ 102, 1297-1307.

50. MacIntosh BR \& Shahi MRS (2011) A peripheral governor regulates muscle contraction. Appl Physiol Nutr Metab 36, $1-11$.

51. Hogan MC, Richardson RS \& Kurdak SS (1994) Initial fall in skeletal-muscle force development during ischemia is related to oxygen availability. J Appl Physiol 77, 2380-2384.

52. Lindstedt SL, McGlothlin T, Percy E, et al. (1998) Task-specific design of skeletal muscle: balancing muscle structural composition. Comp Biochem Physiol B Biochem Mol Biol 120, $35-40$.
53. Infante JP (1987) Docosahexaenoate-containing phospholipids in sarcoplasmic-reticulum and retinal photoreceptors - a proposal for a role in $\mathrm{Ca}^{2+}$-ATPase calcium-transport. Mol Cell Biochem 74, 111-116.

54. Raymer GH, Marsh GD, Kowalchuk JM, et al. (2004) Metabolic effects of induced alkalosis during progressive forearm exercise to fatigue. J Appl Physiol 96, 2050-2056.

55. Sirvent P, Douillard A, Galbes O, et al. (2014) Effects of chronic administration of clenbuterol on contractile properties and calcium homeostasis in rat extensor digitorum longus muscle. PLOS ONE 9, e100281.

56. Hortemo KH, Munkvik M, Lunde PK, et al. (2013) Multiple causes of fatigue during shortening contractions in rat slow twitch skeletal muscle. PLOS ONE 8, e71700.

57. Verburg E, Thorud HMS, Eriksen M, et al. (2001) Muscle contractile properties during intermittent nontetanic stimulation in rat skeletal muscle. Am J Physiol Regul Integr Comp Physiol 281, R1952-R1965.

58. MacIntosh BR, Glumpak JJ, MacNaughton MB, et al. (2011) Pattern of summation with fatigue and inhibition of calcium release in rat muscle. Muscle Nerve 44, 410-417.

59. Pepe S, Tsuchiya N, Lakatta EG, et al. (1999) PUFA and aging modulate cardiac mitochondrial membrane lipid composition and $\mathrm{Ca}^{2+}$ activation of PDH. Am J Physiol 276, H149-H158.

60. Croset M \& Kinsella JE (1989) Changes in phospholipid fattyacid composition of mouse cardiac organelles after feeding graded amounts of docosahexaenoate in presence of highlevels of linoleate - effect on cardiac ATPase activities. Ann Nutr Metab 33, 125-142.

61. Fajardo VA, Bombardier E, Irvine T, et al. (2015) Dietary docosahexaenoic acid supplementation reduces SERCA Ca ${ }^{2+}$ transport efficiency in rat skeletal muscle. Chem Phys Lipids 187, 56-61.

62. McLennan PL, Abeywardena MY, Dallimore JA, et al. (2012) Dietary fish oil preserves cardiac function in the hypertrophied rat heart. BrJ Nutr 108, 645-654.

63. Duda MK, O'Shea KM \& Stanley WC (2009) Omega-3 polyunsaturated fatty acid supplementation for the treatment of heart failure: mechanisms and clinical potential. Cardiovasc Res 84, 33-41. 